Cite this: Phys. Chem. Chem. Phys., 2013, 15, 18835

Received 14th July 2013,

Accepted 19th September 2013

DOI: $10.1039 / \mathrm{c} 3 \mathrm{cp} 52946 \mathrm{k}$

www.rsc.org/pccp

\section{Stabilized gold nanorod-dye conjugates with controlled resonance coupling create bright surface-enhanced resonance Raman nanotags $\dagger$}

\begin{abstract}
Alison McLintock, ${ }^{a}$ Hye Jin Lee ${ }^{b}$ and Alastair W. Wark ${ }^{* a}$
The preparation and characterization of stable and non-aggregated colloidal suspensions of gold nanorodmolecular dye complexes which exhibit very bright surface-enhanced resonance Raman scattering (SERRS) signals is described. A systematic study was performed where both the localized surface plasmon resonance (LSPR) of the nanorod and the molecular resonance of dyes adsorbed onto the rod surface were selectively tuned with respect to the laser excitation wavelengths. Resonance coupling was found to be a significant factor in the overall SERRS enhancement. The polymer stabilized nanorod-dye conjugates were prepared without the added complexity of nanoparticle aggregation as well as having good control over the surface coverage and orientation of the dye molecules. Furthermore, we demonstrate that this new class of Raman nanotags greatly outperforms an approach based on quasi-spherical gold nanoparticles.
\end{abstract}

\section{Introduction}

Understanding and controlling the interaction between the localized surface plasmon resonance (LSPR) of gold and silver nanoparticles and light-absorbing molecular dyes is a rapidly emerging research area. ${ }^{1}$ Hybridization between plasmonic and molecular resonances can give rise to a variety of distinct optical properties which depend on the surface density and assembly behaviour of the dye adsorbed on the nanoparticle surface, the relative spectral overlap of both constituents as well as the distance between the dye layer and nanoparticle surface. A number of experimental ${ }^{2-11}$ and theoretical ${ }^{12-14}$ investigations have been recently reported characterising hybrid dye-metal nanoparticle structures. Of particular interest is the development of nanoparticle-enhanced spectroscopies including surface-enhanced Raman spectroscopy (SERS), ${ }^{15}$ resonance energy transfer, ${ }^{16}$ plasmon-enhanced fluorescence ${ }^{17}$ and fluorescence quenching. ${ }^{18}$

Surface-enhanced resonance Raman scattering, SE(R)RS or SERRS, is a phenomenon that can occur when the laser excitation wavelength, the nanoparticle localized SPR and the

\footnotetext{
${ }^{a}$ Department of Pure and Applied Chemistry, Centre for Molecular Nanometrology, WestCHEM, University of Strathclyde, Glasgow, UK, G1 $1 X L$. E-mail: alastair.wark@strath.ac.uk

${ }^{b}$ Department of Chemistry and Green-Nano Materials Research Centre, Kyungpook National University, 1370 Sankyuk-dong, Buk-gu, Daegu-city, 702-701, Republic of Korea

$\dagger$ Electronic supplementary information (ESI) available: Additional characterization including UV-vis, Raman, SEM, and fluorescence measurements of the dyes and nanorod-dye conjugates as well as more details on the $\left(\mathrm{d} \sigma_{\mathrm{R}} / \mathrm{d} \Omega\right)$ calculation are provided. See DOI: $10.1039 / \mathrm{c} 3 \mathrm{cp} 52946 \mathrm{k}$
}

electronic resonance of the molecular chromophore in contact with the nanoparticle surface all spectrally overlap to some extent. ${ }^{19,20}$ The relative importance of the multiplicative contributions to the overall SERRS signal from both the local electromagnetic field associated with LSPR and the molecular resonance Raman enhancement is an issue that continues to be debated in the literature. In addition, even further enhancement of the Raman signal from colloidal suspensions of gold and silver nanoparticles (NP's) is typically achieved via nanoparticle aggregation. The formation of hot-spots within small clusters of $\mathrm{Ag}$ quasi-spherical nanoparticles has enabled single molecule SERRS measurements to be demonstrated. ${ }^{21}$ However, aggregation also makes profiling the LSPR contributing to the enhanced SERRS response much more complex, with parameters such as cluster size, distribution in size, relative NP orientations and spacing within each cluster, location of probe molecules and changes in particle concentration all important. Consequently, the design of stable colloidal suspensions of bright SERRS-active probes which can be potentially used across a wide range of sensing and imaging applications has continued to be an area of intense research. ${ }^{22}$

In this report, we prepare a series of monodispersed and stable colloidal suspensions of gold nanorod-dye hybrid nanostructures and demonstrate their potential as a new class of bright SERRS-active substrates. A significant advantage of gold nanorods (NR's) is the ability to selectively tune the LSPR excitation across most of the visible and near-infrared (NIR) regions of the electromagnetic spectrum. ${ }^{23}$ In contrast, monodispersed gold quasi-spherical particles are limited to resonance wavelengths of $\sim 510-600 \mathrm{~nm}$ and aggregation is necessary to achieve 
significant SERS enhancements at common laser excitation wavelengths such as 633 and $785 \mathrm{~nm}$. Furthermore, the nanoroddye conjugate system also enables resonance coupling between the rod LSPR and dye to be explored over a range of wavelengths and a small number of studies have recently been reported by Wang et al. ${ }^{9-11}$ and others ${ }^{8,24,25}$ using extinction and fluorescence spectroscopy measurements.

However, even fewer investigations of the SERRS properties of gold nanorod-dye systems have been reported to date. Recently, von Maltzahn et al. ${ }^{26}$ compared a series of NIR dyes via the formation of mixed polyethylene glycol-dye monolayers on the nanorod surface to establish the brightest probe candidate for SERRS imaging in cells at $785 \mathrm{~nm}$. Pal et $a .^{27}$ performed SERRS measurements at $633 \mathrm{~nm}$ on rod aggregates prepared by drop-casting on a glass surface while Cai et al. have modelled the Raman spectrum of 3,3'-diethylthiadicarbocyanine iodide (DTTCI) enhanced by nanorod films. ${ }^{28}$ Gabudean et al. have recently demonstrated the dual use of metal-enhanced fluorescence and SERS on nanorods. ${ }^{29}$ Recently, Murphy et al., reported a SERS study utilizing a dye reporter molecule spaced away from the rod surface via adsorption on a polyelectrolytecoated layer, followed by wrapping with another polymer layer to encapsulate the dye. ${ }^{30}$ They showed that the maximum SERS enhancement is obtained where the plasmon resonance is blueshifted with respect to the single laser excitation wavelength used in their study with optimal signals reported when the LSPR was approximately $50 \mathrm{~nm}$ blue shifted from the laser excitation. This work was also transmission based, rather than a back-scattering configuration, therefore extinction losses along the sample path length are an issue. Also, an initial study by our group ${ }^{31}$ demonstrated that the side-by-side assembly of gold nanorods, which had a NIR dye sequestered into the surfactant bilayer surrounding each nanorod, could be controlled and also resulted in an enhanced SERRS response.

Here, we systematically prepare and characterize different combinations of gold nanorods and dyes whose target resonances strongly overlap at either 633 or $785 \mathrm{~nm}$. As well as controlling factors such as dye surface coverage on the rod surface, the conjugates were wrapped with a polymer layer to achieve long-term stability and signal reproducibility. This enabled comparison between different nanoparticle conjugates and to demonstrate the effective use of the CTAB layer for both sequestering the dye molecules and maintaining their orientation and thus enhanced Raman spectral profile. In particular, the observation of large SERRS intensities while circumventing the need to induce particle aggregation and the generation of interparticle hot-spots represents a model system for promoting investigations of plasmon-dye resonance coupling and in the rational design of SERRS probes.

\section{Experimental}

\subsection{Chemicals}

Cetyltrimethylammonium bromide (CTAB), hydrogen tetrachloroaurate $\left(\mathrm{HAuCl}_{4}\right)$, ascorbic acid, silver nitrate $\left(\mathrm{AgNO}_{3}\right)$, sodium borohydride $\left(\mathrm{NaBH}_{4}\right)$, sodium citrate tribasic dihydrate, 3,3'-diethylthiadicarbocyanine iodide (DTDCI), 3,3'-diethylthiatricarbocyanine iodide (DTTCI), poly(sodium 4-styrenesulfonate) (PSS) $\sim 70 \mathrm{kDa}$, sodium chloride ( $\mathrm{NaCl}$ ), poly(diallyldimethylammonium chloride) (PDDAC), cyclohexane and magnesium sulphate were all purchased from Sigma-Aldrich and used as received. All solutions were prepared using Millipore water.

\subsection{Nanorod synthesis}

Two nanorod solutions were prepared using a modified version of the procedures developed by Sau and Murphy ${ }^{32}$ and Nikoobakht and El-Sayed. ${ }^{33}$ A key difference was that the reaction volume was scaled up to $1 \mathrm{~L}$ to give a large volume of stock NRs to avoid problems associated with batch to batch variations. Furthermore, the samples were centrifuged and resuspended four times in $1 \mathrm{mM}$ CTAB. Each step dilutes the original 0.2 M CTAB stock $\sim 40$-fold on replacing the supernatant following centrifugation such that we assume the CTAB concentration is sufficiently close to $1 \mathrm{mM}$. Establishing a consistent CTAB concentration was critical for the reproducible formation of mixed CTAB-dye layers around the nanorod surface. Samples were characterised using UV-vis measurements to determine the LSPR position, and to calculate the sample concentrations.

\subsection{Preparation of NR-dye conjugates}

$1 \mathrm{mM}$ DTTCI and DTDCI stock solutions were prepared in $\mathrm{MeOH}$, and further diluted in water immediately prior to addition of the desired volume of dye to $6 \mathrm{ml}$ of stock NR solution to give a bulk dye concentration of $0.5,1,5,10,20$ or $50 \mu \mathrm{M}$ and the samples were left to equilibrate overnight ( $\sim 16$ hours) at $27{ }^{\circ} \mathrm{C}$. Next, PSS coating of the conjugates was carried out by adding $1.2 \mathrm{ml}$ of $10 \mathrm{mg} \mathrm{ml}^{-1}$ PSS in $5 \mathrm{mM} \mathrm{NaCl}$ dropwise to the NR-dye conjugate solution while stirring rapidly. After a few minutes the samples were then centrifuged at $\sim 4774 \mathrm{~g}$ for $20 \mathrm{~min}$ and resuspended in $6 \mathrm{ml}$ of water; this was repeated a further three times to remove excess bulk dye and CTAB molecules. Additional characterization of the particle concentrations and aggregation state of the NR-dye conjugate concentrations was performed using a NanoSight LM20 and accompanying Nanoparticle Tracking Analysis software as well as UV-vis spectroscopy.

\subsection{Preparation of spherical gold nanoparticles}

Gold nanoparticles were prepared by a variation of the Turkevich method. ${ }^{34}$ Here, $100 \mathrm{mg}$ of $\mathrm{HAuCl}_{4}$ was added to $490 \mathrm{ml}$ water, heated to boiling and then reduced using $105.7 \mathrm{mg}$ of sodium citrate tribasic dihydrate in $10 \mathrm{ml}$ water to afford colloidal gold with an average diameter of approximately $38 \mathrm{~nm}$. An extinction coefficient of $7.8 \times 10^{9} \mathrm{M} \mathrm{cm}^{-1}$ was used to calculate the nanoparticle concentration. ${ }^{35}$

\subsection{SERRS measurements}

All SERRS measurements were performed using a Renishaw InVia Raman inverted microscope system, equipped with a $20 \times$ long working distance objective. Two excitation sources were used at $632.8 \mathrm{~nm}$ and $785 \mathrm{~nm}$ along with an 1800 or 1200 gr per mm grating respectively. The typical laser power at 
$633 \mathrm{~nm}$ was $1.0 \mathrm{~mW}$ while for $785 \mathrm{~nm}$ laser powers of $12.0 \mathrm{~mW}$ and $1.2 \mathrm{~mW}$ were used depending on the nanotag signal intensity obtained. Specifically, $12 \mathrm{~mW}$ was utilised at conjugates prepared using dye concentrations of $10 \mu \mathrm{M}$ and below while $1.2 \mathrm{~mW}$ was used at higher concentrations. Also, sample spectra acquired at both laser powers and normalized with respect to a cyclohexane reference under identical conditions could be directly compared. Samples were analysed through transparent bottom micro-titre plates with $300 \mu \mathrm{l}$ of the nanorod conjugate solution placed in each well. A signal collection time of $10 \mathrm{~s}$ per spectra was used. Cyclohexane was used to optimise the signal collection as well as to provide an intensity reference for data normalization. For each spectrum reported, a minimum of three sample aliquots in separate wells were acquired and compared. For experiments involving the controlled aggregation of quasi-spherical nanoparticles $1 \mu \mathrm{l}$ of $1 \mathrm{M} \mathrm{MgSO}_{4}$ was added to $500 \mu \mathrm{l}$ of colloid followed by mixing. All spectra were processed and background corrected using Grams/AI software (version 7.0).

\subsection{Scanning electron microscopy (SEM)}

SEM images were obtained using an FEI Sirion 20 ultra-high resolution Schottky field emission scanning electron microscope with FEI software. Further details and representative images are provided in the ESI. $\dagger$

\section{Results and discussion}

\subsection{Design and preparation of nanorod-dye conjugates}

Gold nanorod (NR) sizes and dyes were selected which had resonances closely matching one of two common Raman excitation wavelengths (633 and $785 \mathrm{~nm}$ ) and which were then selectively combined to create a range of NR-dye conjugates. An outline of the strategy used for the preparation of stable and SERRS-active NR-dye conjugates is shown in Fig. 1(a). The bilayer of CTAB surrounding the nanorod is responsible for both directing the rod shape during synthesis and maintaining colloidal stability. However, the presence of this bilayer also complicates subsequent surface functionalization, and this has previously hindered the use of nanorods for SERS-based applications. Furthermore, CTAB has a fairly weak affinity for the gold $\langle 110\rangle$ longitudinal face and a net desorption from the rod surface starts to occur when the bulk concentration is lowered below $\sim 0.5 \mathrm{mM}$, which results in colloidal instability when cleaning. Consequently, the CTAB concentration in the prepared nanorod stock solutions was fixed at $1 \mathrm{mM}$ by performing a minimum of four repeat centrifuge and resuspension (in $1 \mathrm{mM} \mathrm{CTAB}$ ) steps after the NR synthesis.

Following the preparation of the nanorod stock solutions, the first step in Fig. 1(a) shows the addition of a reporter dye, either DTDCI (Fig. 1(e)) or DTTCI (Fig. 1(f)), to form a mixed layer of CTAB and dye. Instead of replacing the CTAB, both our group $^{31}$ and another ${ }^{36}$ have shown that hydrophobic molecules can be sequestered into the CTAB bilayer with high efficiencies. A further advantage of maintaining the CTAB bilayer is that it controls the orientation of the dye with respect to the NR surface, even at very low dye fractional surface coverages. The second preparative step involves wrapping the positively charged NR-dye assembly with a polyelectrolyte layer of PSS. The polymer wrapping has a number of roles: it encapsulates the conjugate and enables the subsequent removal of any excess dye and CTAB from the bulk solution, and also stabilises the conjugate, (a)
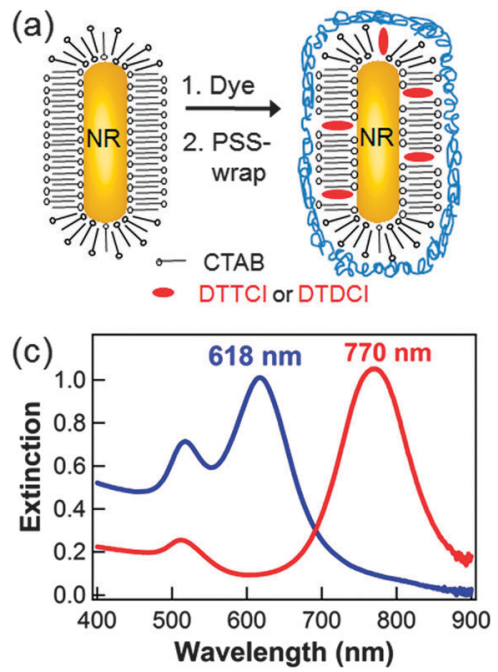

(e)

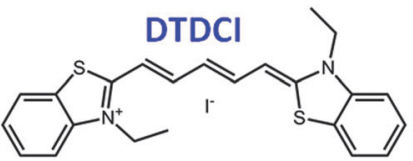

(b)
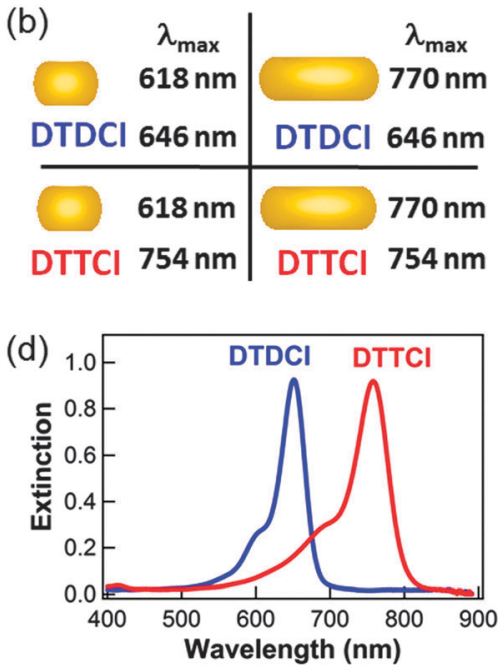

(f)

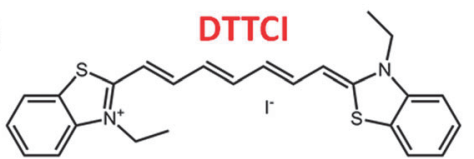

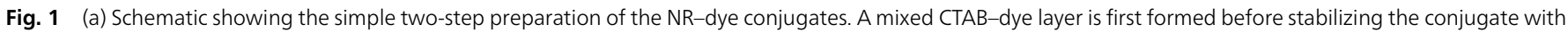

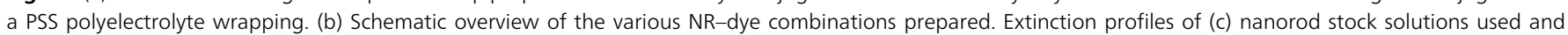
(d) $5 \mu \mathrm{M} \mathrm{DTDCI}$ and DTTCI in MeOH. The chemical structures of the (e) DTDCI and (f) DTTCI dyes are also shown. 
resulting in reproducible SERRS signals when comparing repeat preparations as well as after several months' storage.

Different combinations of nanorods and dyes were compared as summarised in Fig. 1(b) to observe and quantify the effect of altering the nanorod plasmon resonance and the dye molecular resonance. Fig. 1(c) and (d) show the extinction spectra for the NR and dye stock solutions used respectively. The first NR stock solution featured transverse and longitudinal plasmon peaks at $516 \mathrm{~nm}$ and $618 \mathrm{~nm}$ respectively, along with an average rod length of $16 \mathrm{~nm}$ and an aspect ratio of 2.0. The second NR sample had transverse and longitudinal peaks at $511 \mathrm{~nm}$ and $770 \mathrm{~nm}$ respectively, for which an average length of $47 \mathrm{~nm}$ and an aspect ratio of 4.3 were measured. For both stock solutions, the particle concentration was fixed at $\sim 1 \mathrm{nM}$ based on extinction coefficients of $2.5 \times 10^{9}$ and $4.6 \times 10^{9} \mathrm{M}^{-1} \mathrm{~cm}^{-1}$ respectively at the $\lambda_{\text {max }}$ of the longitudinal LSPR. ${ }^{37}$ The choice of DTDCI and DTTCI as Raman reporter molecules was based on the fact that they are both strongly absorbing $\left(\lambda_{\max }=646 \mathrm{~nm}\right.$ and $754 \mathrm{~nm}$ respectively) close to the 633 and $785 \mathrm{~nm}$ laser excitation wavelengths. In addition, both dyes are structurally very similar differing only in the length of the polymethine chain and have poor water solubility.

The formation of a mixed CTAB-dye layer is supported by several experimental observations. These include comparing the improved stability of the dye absorbance spectrum in $1 \mathrm{mM}$ CTAB solution compared to water only where the dye molecules form aggregates (see Fig. S2, ESI $\dagger$ ). In addition, fluorescence spectroscopy (Fig. S3a and b, ESI $\dagger$ ) shows quenching quickly occurring upon the addition of dye to the NR solutions compared to when nanorods are absent. The large Raman signals described later support the adsorption of dye molecules onto the nanorod surface, and with the bulk CTAB concentration fixed at $1 \mathrm{mM}$ no destabilization of the colloid solution was observed at dye concentrations as high as $50 \mu \mathrm{M}$. When preparing the conjugates, each sample was left overnight to reach a steady-state fractional surface coverage of dye prior to PSS wrapping.

Considerable effort was made during the preparation of the NR-dye conjugates to ensure that no significant level of particle aggregation took place following the addition of the dyes and in the final conjugate solution as even a small fraction of aggregates can potentially have a significant impact on the bulk SERRS intensities obtained, as well as cause a shift in the nanorod LSPR. ${ }^{31}$ Also, in order to quantitatively compare different conjugate solutions, the particle concentrations were kept constant throughout the preparation and analysis processes. A number of complementary techniques were utilised to determine whether or not aggregates were present and to monitor the particle concentrations of the samples, including the analysis of extinction spectra, SEM (see Fig. S1, ESI $\dagger$ ) and also the application of NanoSight video tracking analysis. ${ }^{38}$

\subsection{Observation of resonance coupling}

A series of NR-dye conjugates were prepared where both the NR and the dye have overlapping resonances, or the NR and dye have different resonances. A fixed aliquot volume taken from

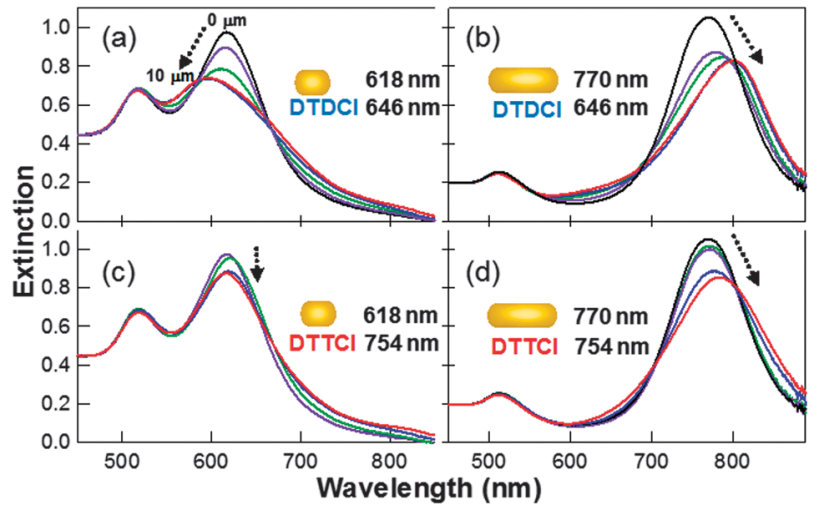

Fig. 2 Extinction spectra of PSS-wrapped nanorod-dye conjugates prepared at a number of dye concentrations $(0,0.5,1,5,10 \mu \mathrm{M})$ and a fixed nanorod concentration (arrow indicates direction of longitudinal plasmon resonance shift and also increasing dye concentration). (a) $618 \mathrm{~nm} \mathrm{NR}$ with DTDCl; (b) $770 \mathrm{~nm} \mathrm{NR}$ with DTDCl; (c) $618 \mathrm{~nm}$ NR with DTTCl; and (d) $770 \mathrm{~nm} \mathrm{NR}$ with DTTCl.

the same nanorod stock solutions was used for each preparation and to which a range of bulk dye concentrations $(0.5-50 \mu \mathrm{M})$ were added and left overnight. The conjugates were then polymer wrapped followed by centrifugation and resuspension $(3 \times)$ in water to ensure that excess bulk dye is removed from the solution and then characterized using UV-vis spectroscopy, with the results shown in Fig. 2. This approach ensures that there is no contribution from any remaining free dye whose concentration will be at least two orders of magnitude lower than the UV-vis detection limit of $\sim 0.5 \mu \mathrm{M}$, and that the spectral shift is due to dye adsorbed onto the nanorod surface. All four combinations of nanorods and dyes resulted in both dampening and a spectral shift of the longitudinal plasmon peak, with both effects increasing at higher dye concentrations. It can be seen in Fig. 2 that the relative positions of the dye and rod resonances are important. Both the size and direction of shift, either to the blue or red, depends on both the relative absorbance wavelengths of the dye and the nanorod.

The size and direction of the longitudinal LSPR shift varies depending on the NR-dye combination in question. When DTTCI is adsorbed onto the $618 \mathrm{~nm}$ NR sample, the longitudinal plasmon undergoes a small red shift of $\sim 3 \mathrm{~nm}$. When the same dye is adsorbed onto a $770 \mathrm{~nm}$ NR sample, the shift direction remains the same, but the size of shift increases up to approximately $20 \mathrm{~nm}$ for a $50 \mu \mathrm{M}$ bulk dye concentration. DTDCI displays very different behaviour when it is adsorbed onto $618 \mathrm{~nm}$ NRs, the plasmon undergoes a blue shift of up to $24 \mathrm{~nm}$. When adsorbed onto a $770 \mathrm{~nm} \mathrm{NR}$, the direction of shift changes to the red, and is still significant, at up to around $32 \mathrm{~nm}$. In all cases, the amount of shift has been shown to be dependent upon the bulk dye concentration, with increased dye surface coverage resulting in increased plasmon shift and increased dampening of the signal. Similar trends have been reported by Ni et al. ${ }^{10}$ who reported a net blue shift in the longitudinal plasmon peak position when the $\lambda_{\max }$ of the adsorbing dye was greater than the longitudinal plasmon peak position, and a net red shift when the initial longitudinal 
plasmon maximum was greater than the absorbance of the adsorbing dye.

A significant difference in our results compared to several recent studies on metal nanoparticle-dye systems is that no Rabi splitting was observed where the LSPR band develops into two bands. This effect appears to be associated with instances where dye molecule aggregates (which support excitons) are interacting with the nanoparticle surface, and is most dramatic where the LSPR and molecular resonance have the biggest overlap and also at larger dye fractional surface coverages. ${ }^{39-41}$ The absence of plasmon band splitting observed here despite a larger bulk excess of dye molecules is attributed to differences in control over the dye assembly around the nanorod involving a relatively uniform dispersion of non-aggregated single dye molecules. Maintaining a stable CTAB bilayer around each rod limits the extent to which both the DTTCI and DTDCI dyes used here assemble into J-aggregates. In pure water, both dyes quickly selfassemble, while in $1 \mathrm{mM}$ CTAB solution the dye absorbance spectra are much more stable (see Fig. S2, ESI + ). The observation of "weaker" dye-nanoparticle resonance coupling (i.e. smaller LSPR shifts with no plasmon band splitting) has also been reported $^{3,42}$ in contrast to studies where the molecular stacking and orientation of the dye molecules on the metal nanoparticle surface is relatively uncontrolled ${ }^{10,39,40}$ resulting in "strong" exciton-plasmon coupling (i.e. larger LSPR shifts and band splitting). In this initial study we have focused on performing a quantitative SERRS study where the CTAB bilayer provides good control over the dye behaviour on the nanorod surface.

\subsection{SERRS analysis}

The SERRS signals of the different combinations of dyes and nanorods summarized previously in Fig. 1(b) were then systematically acquired at both 633 and $785 \mathrm{~nm}$ laser excitation frequencies. In each case, a fixed volume of the nanorod stock solution was exposed to dye concentrations ranging from 0.5-50 $\mu \mathrm{M}$ before polymer wrapping and subsequent removal of excess bulk dye, CTAB and PSS via repeat centrifugation and resuspension. A combination of extinction spectral monitoring, SEM and dynamic nanoparticle imaging and tracking measurements were performed for each of the samples to ensure that the nanorods were monodispersed with negligible levels of particle aggregation. Furthermore, the intensities of the SERRS spectra reported were normalized with respect to a cyclohexane reference acquired under identical conditions (i.e. laser power and focus, sample volume, integration time) as the sample measurement. This was to promote a direct comparison of spectral intensities acquired between samples at different laser excitation wavelengths and laser intensities.

Fig. 3 clearly shows that the SERRS response increases when higher bulk concentrations of DTTCI dye are utilized to prepare the PSS-wrapped nanorod-dye conjugates due to an increase in the number of dye molecules encapsulated in the CTAB bilayer. At bulk dye concentrations above $\sim 20 \mu \mathrm{M}$, further changes in the SERRS intensity are much less which suggests that a maximum dye loading has been achieved at the steady-state conditions employed. In addition, Fig. 3 shows that the relative

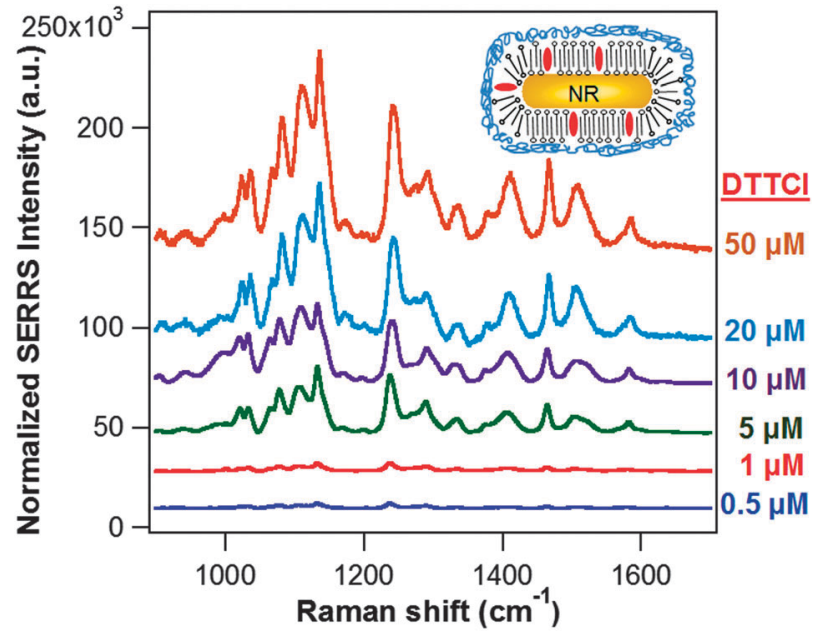

Fig. 3 SERRS spectra obtained for a series of PSS-wrapped rod-dye conjugates prepared using a fixed concentration of NR's $\left(\lambda_{\max }=770 \mathrm{~nm}\right)$ and various concentrations of DTTCl dye. A bulk CTAB concentration of $1 \mathrm{mM}$ is also maintained throughout. Laser excitation wavelength $=785 \mathrm{~nm}$. The spectra have been vertically offset to allow comparison.

peak intensities in the spectral profile did not change as a function of dye concentration. This suggests the dye orientation on the nanorod surface is maintained by the CTAB bilayer unlike the data shown later (in Fig. 6) where the spectral profile changes as a function of dye surface coverage in the absence of CTAB. It is also important to point out that no difference in the spectral profile was observed when comparing suspensions before or after PSS wrapping.

The change in SERRS signal as a function of bulk dye concentration was found to fit a Langmuir adsorption isotherm (see Fig. S5a, ESI $\dagger$ ) which gave an equilibrium affinity constant of $K_{\mathrm{ads}}=1.07( \pm 0.1) \times 10^{5} \mathrm{M}^{-1}$. This is about an order of magnitude higher than the value obtained by Alkilany et al. for the partitioning of 1-napthol into the CTAB bilayer. ${ }^{36}$ Ideally, it would be preferable to also measure the ratio of dye molecules free in the bulk solution to that adsorbed on the nanorod surface using UV-vis spectroscopy, however we found that the tendency of the free dye molecules to aggregate over longer periods of time, especially for DTTCI, prevented sufficiently accurate measurements (see Fig. S2 in the ESI $\dagger$ ). Repeat measurements where the DTDCI dye is instead loaded onto nanorods from the same stock solution $\left(\lambda_{\max }=770 \mathrm{~nm}\right)$ are also shown in the ESI $\dagger$ (Fig. S4 and S5b) indicating similar concentration dependent behavior was also observed.

A comparison of the normalized SERRS intensities acquired for different nanorod-dye combinations is summarized in Fig. 4 and 5, which allowed a number of observations to be made. Each sample was analyzed at both 633 and $785 \mathrm{~nm}$ excitation and the bulk dye concentration was fixed at $5 \mu \mathrm{M}$ during the rod-dye conjugate preparation. In order to quantitatively compare the relative SERRS intensities for the different combinations, Fig. 5 summarizes different selected peak intensity values for each of the spectra shown in Fig. 4 . The values were obtained from peak maxima and minima intensity positions 
(described in the Fig. 5 caption) extracted prior to baseline correction and used as a numerical indicator with which relative SERRS intensities could be compared.

Control measurements acquired in the absence of dye molecules for both the stock solution nanorods in $1 \mathrm{mM} \mathrm{CTAB}$ and for PSS-wrapped rods are provided in the ESI, $\uparrow$ Fig. S7. Using identical laser powers, integration times and particle concentrations it can be clearly seen that both CTAB and PSS have a negligible contribution to the SERRS spectral profiles shown in Fig. 3-5. Also shown in the ESI $\dagger$ in Fig. S8 are resonance Raman spectra for both the DTDCI and DTTCI dyes deposited onto a planar gold film to quench the fluorescent background signal. Significantly greater laser powers and accumulation times were required than for the solution-based measurements. For DTDCI, no resonance Raman spectrum was obtained at $785 \mathrm{~nm}$, only at $633 \mathrm{~nm}$, while for DTTCI resonance Raman spectra at both excitation wavelengths were observed. The poorer signal-to-noise for the resonance Raman measurements indicate that the overall SERRS enhancement is due to a combination of molecular and plasmon resonance contributions.

A. Changing laser wavelength. It is expected that the largest SERRS enhancements will be obtained when both the dye and nanorod resonances overlap with the excitation wavelength. ${ }^{19,43}$ This is clearly seen in Fig. 4(a) where both the DTDCI dye and nanorod resonances overlap with the $633 \mathrm{~nm}$ laser resulting in a SERRS intensity $\sim 2.7$ times greater than the normalized signal obtained when the measurement is repeated at $785 \mathrm{~nm}$ excitation. In Fig. 4(d) and 5(d), where both the DTTCI dye and nanorod resonances predominantly overlap with the $785 \mathrm{~nm}$ laser, the relative differences in normalized SERRS signals between 633 and $785 \mathrm{~nm}$ excitation is even greater, about 25-fold higher at $785 \mathrm{~nm}$. The larger contrast observed between the two different SERRS measurements in (d) compared to the smaller difference between the two spectra in

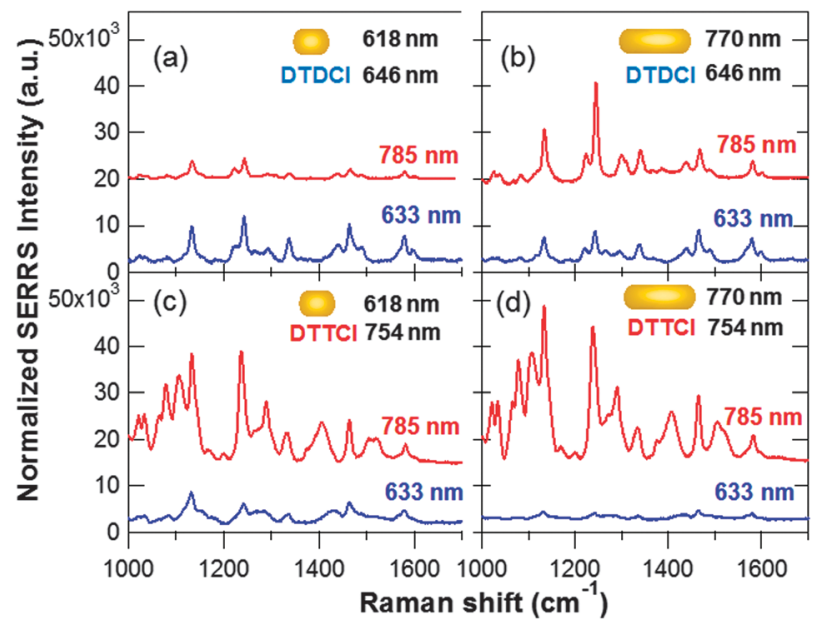

Fig. 4 Normalized SERRS spectra obtained at an excitation wavelength of $785 \mathrm{~nm}$ and $633 \mathrm{~nm}$. All the data shown is for conjugates prepared using a $5 \mu \mathrm{M}$ bulk dye concentration: (a) $618 \mathrm{~nm} \mathrm{NR}, \mathrm{DTDCl}$; (b) $770 \mathrm{~nm} \mathrm{NR}$, DTDCl; (c) $618 \mathrm{~nm} \mathrm{NR}, \mathrm{DTTCl}$; and (d) $770 \mathrm{~nm} \mathrm{NR}$, DTTCl. Each spectrum is normalized with respect to a cyclohexane standard and baseline corrected.

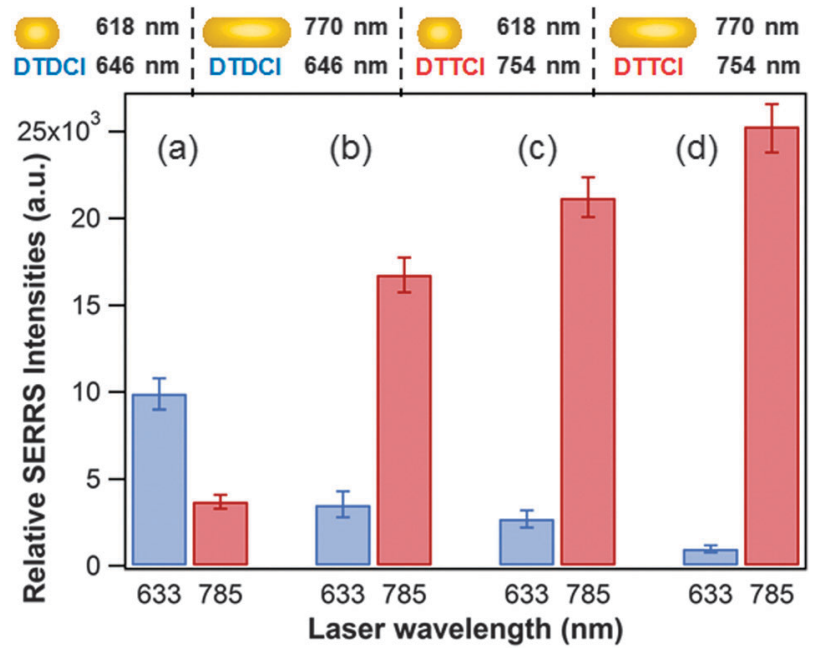

Fig. 5 Comparison of normalized SERRS intensities for the corresponding spectra and excitation wavelengths showed previously in Fig. 4(a)-(d). The intensity of a representative peak was obtained by finding the maximum peak height at $1245( \pm 2) \mathrm{cm}^{-1}$ and subtracting the minimum at $1195( \pm 5) \mathrm{cm}^{-1}$ for DTDCI. The process was repeated for DTTCI using the maximum peak height at $1241( \pm 2) \mathrm{cm}^{-1}$ and subtracting the minimum peak height at $1195( \pm 5) \mathrm{cm}^{-1}$.

(a) is different from what would be expected based on each dyes absorption spectrum shown in Fig. 1. The DTTCI dye has a broader absorption spectrum partially overlapping with both laser wavelengths while DTDCI has very little overlap at $785 \mathrm{~nm}$ excitation. However, significantly different extinction behavior was observed in Fig. 2 for both systems with the LSPR peak in (a) blue-shifting further away from $633 \mathrm{~nm}$ while in (d) a redshift closer to $785 \mathrm{~nm}$ occurs on dye adsorption.

The changes in SERRS intensities observed on switching between 633 and $785 \mathrm{~nm}$ excitation were observed consistently for conjugates prepared at all bulk dye concentrations (1-50 $\mu \mathrm{M})$. Thus, with the exception of the rod-dye combination in sample (a), the relative SERRS intensities obtained for $785 \mathrm{~nm}$ excitation were consistently larger than that obtained at $633 \mathrm{~nm}$ excitation for samples (b-d).

B. Changing the dye resonance. Information on the effect of changing the molecular dye resonance on the SERRS intensity can be obtained from the spectral pairs in Fig. 4(a) and (c) where the rod-dye conjugates were prepared from the same stock solution of nanorods $\left(\lambda_{\max }=618 \mathrm{~nm}\right)$. At $633 \mathrm{~nm}$ excitation, the SERRS signal is $\sim 3.5 \times$ higher for the DTDCI dye in Fig. 4 (a) than the DTTCI dye in Fig. 4(c) as expected due to the overlap in the dye and laser wavelengths. Similarly, at $785 \mathrm{~nm}$ excitation, the SERRS signal associated with the DTTCI dye in Fig. 4(c) is more than 5-fold greater than the corresponding measurement in Fig. 4(a) and also larger than the DTDCI signal at $633 \mathrm{~nm}$ where the rods and dye are both resonant with the incident laser. This may in part be due to slight differences in fractional surface coverage between the DTTCI and DTDCI dyes on the nanorods.

A similar comparison can also be made between Fig. 5(b) and (d) involving the larger aspect ratio nanorods $\left(\lambda_{\max }=\right.$ $770 \mathrm{~nm}$ ). For both dyes, the spectral intensities were greater at $785 \mathrm{~nm}$ laser excitation than $633 \mathrm{~nm}$, even for DTDCI. 
This initially suggests that the rod LSPR is playing a slightly more dominant role in the overall SERRS enhancement than the dye. On the other hand, when comparing (c) and (d) in Fig. 4 and 5 , the DTTCI signal from the short rod (off-resonance) is nearly the same as the longer rod (on-resonance) which strongly suggests the resonance contribution of the dye is the stronger effect. However, it is important to point out that comparing (b) and (d) with different rod sizes (stock solution concentrations are similar) involves differences in available surface areas and a comparison between (c) and (d) is more accurate since they were prepared from the same rod solution.

Ultimately, understanding of the relative importance of the dye and rod contributions to the overall SERRS signal also depends on knowing the distribution of dye molecules between the rod ends and sides and if this changes as a function of dye fractional surface coverage. The LSPR electromagnetic field enhancements are greatest at the rod ends, rather than along the particle sides ${ }^{44}$ and thus dye molecules located at the ends will have a relatively larger contribution to the SERRS intensities, however, the CTAB layer at the rod ends has a lower molecular density and this will affect the dye adsorption in this region since the strong surface affinity of the dye for the rod surface is based on the CTAB bilayer. Further work is underway involving a study at the single particle level including control of the incident light polarization parallel and perpendicular to both rod axes and will be reported in a future publication.

C. Quantification of SERRS intensities. Because both dyes used in this study are strongly fluorescent, the determination of an absolute SERRS enhancement factor was not possible. ${ }^{45,46}$ Instead, we have focused on performing a relative study between different dye-rod combinations and a comparison between nanorod and quasi-spherical nanoparticles is also discussed in the next section. Furthermore, comparison of the data in Fig. 4 clearly shows that even though DTDCI and DTTCI are structurally very similar, the SERRS spectra are significantly different (see Fig. S6 in the ESI $†$ for extended spectra). Since all the SERRS data presented are normalized with respect to the signal from a neat solution of cyclohexane acquired under identical conditions, we instead calculated the differential Raman cross-section $\left(\mathrm{d} \sigma_{\mathrm{R}} / \mathrm{d} \Omega\right.$ ) focusing on the two dye-rod combinations (a) and (d) at excitation wavelengths of 633 and $785 \mathrm{~nm}$ where the brightest signal was obtained. Details of the calculation are provided in the ESI. $\dagger$ For DTTCI-rod $\left(\lambda_{\max }=\right.$ $770 \mathrm{~nm})$ conjugates prepared at a dye concentration of $50 \mu \mathrm{M}$ (corresponding to the largest SERRS signal in Fig. 3) a $\left(\mathrm{d} \sigma_{\mathrm{R}} / \mathrm{d} \Omega\right.$ ) of $\sim 3.9 \times 10^{-20} \mathrm{~cm}^{2} \mathrm{sr}^{-1}$ was obtained. Similarly, for the DTDCI $(50 \mu \mathrm{M})-\operatorname{rods}\left(\lambda_{\max }=618 \mathrm{~nm}\right)$ combination which resulted in the highest SERRS intensity at $633 \mathrm{~nm}$ excitation, a $\left(\mathrm{d} \sigma_{\mathrm{R}} / \mathrm{d} \Omega\right)$ of $\sim 1.4 \times 10^{-20} \mathrm{~cm}^{2} \mathrm{sr}^{-1}$ was determined. These results also reflect the $\sim 2.5$-fold difference in intensities observed between the relevant intensities in Fig. 5(a) and (d) at $633 \mathrm{~nm}$ and $785 \mathrm{~nm}$ respectively for conjugates prepared at a different bulk concentration.

The calculated differential cross-section values are comparable with the brightest signals obtained in the recent study by Amendola and Meneghetti ${ }^{46}$ who quantified the SERS response for a range of spherical nanoparticle, dye-label combinations and which also contained a significant subpopulation of aggregated nanoparticles. To gain further insight, a direct comparison of the relative brightness of the nanorod-dye conjugates versus labelled spherical nanoparticles is described in the next section.

D. Comparison of nanorod versus spherical shapes. Since the majority of SERS studies performed to-date have focused on the use of colloidal solutions of quasi-spherical metal nanoparticles, we next compared the relative SERRS response of the nanorod-dye conjugates with gold nanoparticles prepared by the well-established citrate reduction method. A stock solution with an average diameter of $38 \mathrm{~nm}$ was prepared (see Fig. S9 in the ESI $\dagger$ for SEM image). Instead of creating a mixed CTABdye bilayer on the surface of the spherical particles, where the molecular density and bilayer organization is likely to be significantly different between the rod and spherical morphologies, we adopted an approach where the positively charged DTTCI was directly introduced to the negatively charged citratestabilized nanoparticle solution in a manner comparable with previous investigations. The aim was to simply establish the maximum SERRS signal for the quasi-spherical nanoparticledye system (which would not be obtained in a mixed monolayer system) and compare signal levels with that obtained for the maximum signals obtained for the monodispersed nanorodDTTCI conjugates described earlier in Fig. 3. This approach also allowed some insight into the importance of the CTAB bilayer in controlling the dye orientation with respect to the nanoparticle surface.

Fig. 6 compares normalized SERRS spectra obtained at $785 \mathrm{~nm}$ excitation for polymer-wrapped DTTCI-rod conjugates and quasi-spherical nanoparticles. When DTTCI was introduced to the citrate-stabilized Au colloid stock solution, nanoparticle aggregation started to occur at bulk dye concentrations above

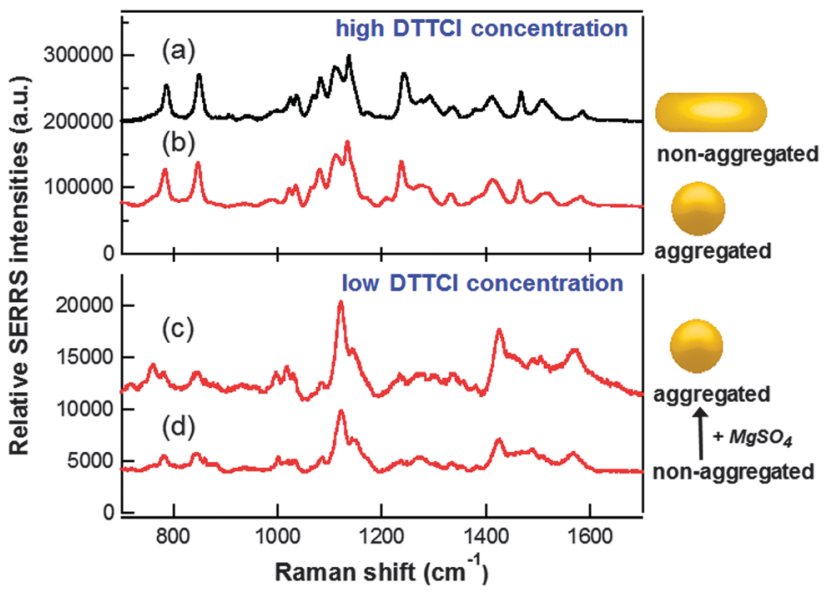

Fig. 6 Normalized SERRS spectra at $785 \mathrm{~nm}$ excitation for (a) monodispersed solution of polymer-wrapped DTTCl-rod conjugates prepared with a $50 \mu \mathrm{M}$ bulk dye concentration; (b) aggregated quasi-spherical $\mathrm{Au}$ nanoparticles in the presence of $2 \mu \mathrm{M} \mathrm{DTTCl}$ and $\mathrm{MgSO}_{4}$, representing the maximum SERRS obtained from this system. Measurements at a lower DTTCl concentration of $0.05 \mu \mathrm{M}$ are shown for quasi-spherical $\mathrm{Au}$ particles (c) following aggregation induced by $\mathrm{MgSO}_{4}$ and (d) non-aggregated. 
$\sim 0.05 \mu \mathrm{M}$ (see Fig. S10 in the ESI $\dagger$ ). Therefore, in order to first compare the signal from non-aggregated spherical particles, Fig. 6(d) shows the SERRS spectrum obtained at a bulk dye concentration of $0.05 \mu \mathrm{M}$. When an optimal amount of $\mathrm{MgSO}_{4}$ aggregating agent was added to this solution to maximise the SERRS enhancement, a relatively modest $\sim 2$-fold increase in intensity was observed (see Fig. 6(c)). Comparison of the spectra in (c) and (d) show similar peak profiles, both of which are markedly different from the spectra obtained for the nanorod-dye complexes. However, when higher DTTCI concentrations are introduced to the quasi-spherical nanoparticle colloid, the resulting SERRS profile was very similar to that of the rod-dye conjugates (compare Fig. 6(a) and (b)). This emphasizes the role of the CTAB bilayer in controlling the orientation of the dye on the nanorod surface while the average orientation of the dye on the citrate-stabilized quasi-spherical particles must vary with dye fractional surface coverage. The relationship between the molecule orientation on a nanoparticle surface and the SERS spectral profile has been described elsewhere. ${ }^{47,48}$

The SERRS spectrum of the polymer-wrapped dye-rod conjugates in Fig. 6(a) represents the maximum signal obtained at a fixed nanorod concentration of $\sim 1.1 \mathrm{nM}$ with no nanoparticle aggregation. The most intense peak in (a) is $\sim 24$ fold greater than the most intense peak obtained for the non-aggregated quasi-spherical particles in Fig. 6(d). The role of nanoparticle aggregation to increase SERRS signals is well-established, and the spectrum in Fig. 6(b) represents the maximum response obtained following a series of repeat measurements involving the aggregation of the quasi-spherical nanoparticles at higher DTTCI concentrations (see Fig. S11 in the ESI $\dagger$ for additional spectra). Comparing (a) and (b), the signal for the non-aggregated rods is approximately $10 \%$ higher than for the aggregated spheres. The concentrations of the nanorod $(\sim 1.1 \mathrm{nM})$ and quasi-spherical $(\sim 0.26 \mathrm{nM})$ stock solutions are not identical while the surface area of the average spherical particle is $\sim 1.8 \times$ greater than that of the nanorods. It is also reasonable to assume a significantly higher dye fractional coverage on the citratestabilized spheres than the CTAB-coated rods. Thus the difference in the total number of dye molecules adsorbed onto a nanoparticle surface in both cases will be considerably less than a factor of two. Given that inducing aggregation results in orders of magnitude increase in the SERRS response of spherical particles ${ }^{38}$ and the fact that the normalized SERRS signal associated with the monodispersed nanorod-dye conjugates is comparable with the aggregated spherical system provides evidence that the nanorod-dye conjugates are significantly brighter. Recently, we have shown that the controlled side-by-side assembly of the nanorod-dye conjugates can result in up to a $\sim 4$-fold increase in signal. ${ }^{31}$ Furthermore, when rod aggregation is performed in a random fashion in the presence of DTTCI, then an even larger increase in intensity is easily achieved, such as the example shown in Fig. S12 in the ESI. $†$

An absolute comparison between spheres and rods is difficult to achieve due to the complexity associated with factors such as dye surface coverage, orientation, available surface area and particle size distribution. However, these results clearly demonstrate nanorod-dye assemblies to be extremely promising SERRS substrates compared to more conventional spherical colloidal particles of similar dimensions. In the future, it will be interesting to compare the nanorod-dye system quantitatively with other single encoded SERS particles which typically use thiol-PEG and/or silica coatings for encapsulation of reporter molecules. ${ }^{49}$

\section{Conclusion}

A series of stable, monodisperse suspensions of polymer-wrapped gold nanorod-dye conjugates were prepared and their optical extinction and SERRS properties characterized by selectively tuning both the plasmon resonance of the nanorod and the molecular resonance of the dye with respect to the laser excitation wavelengths (633 $\mathrm{nm}$ and $785 \mathrm{~nm}$ ). A particular advantage of our approach is its simplicity with bright Raman signals obtained without nanorod aggregation and good control over the surface coverage and orientation of reporter molecules on the nanorod surface. In comparison, most efforts at producing SERS probes have involved an aggregation step during their preparation which is relatively uncontrolled ${ }^{38,50,51}$ with a recent trend in the literature towards achieving greater aggregation control as part of the SERS optimization process. ${ }^{52,53}$ Further static and dynamic studies at the single nanoparticle level are currently underway to investigate the polarization dependence of the SERRS response and assess if there is a preferential adsorbance of dye, at least initially, on the rod ends or sides. This will also let us assess the percentage of particles that are SERRS active and compare with other substrates such as nanostars, ${ }^{54}$ capsules $^{55,56}$ and aggregated systems. We have clearly shown the nanorod-dye platform to be a highly promising class of SERRS labels that offers a number of advantages. The polymer-wrapped conjugates were found to be very stable, allowing a significant Raman signal to be obtained after months of storage. The use of polyelectrolyte layers both as a platform for subsequent biofunctionalization ${ }^{57}$ and to also significantly reduce the cell toxicity of CTAB-coated nanorods ${ }^{58}$ has also been demonstrated. In particular, we hope the simplicity of the system will further promote the rational design of SERRS substrates for use in a wide range of applications.

\section{Acknowledgements}

This work was supported by an Engineering Physical Sciences Research Council (EPSRC) Science and Innovation Award in Nanometrology and an EPSRC First Grant award (\#EP/H030468/ 1). We would also like to thank Prof. WE Smith for valuable discussions over the course of the work.

\section{References}

1 H. Chen, T. Ming, L. Zhao, F. Wang, L. D. Sun, J. Wang and C. H. Yan, Nano Today, 2010, 5, 494-505.

2 N. Kometani, M. Tsubonishi, T. Fujita, K. Asami and Y. Yonezawa, Langmuir, 2001, 17, 578-580.

3 A. J. Haes, S. Zou, J. Zhao, G. C. Schatz and R. P. Van Duyne, J. Am. Chem. Soc., 2006, 128, 10905-10914.

4 J. Zhao, L. Jensen, J. Sung, S. Zou, G. C. Schatz and R. P. Van Duyne, J. Am. Chem. Soc., 2007, 129, 7647-7656.

5 G. P. Wiederrecht, G. A. Wurtz and J. Hranisavljevic, Nano Lett., 2004, 4, 2121-2125. 
6 I. I. S. Lim, F. Goroleski, D. Mott, N. Kariuki, W. Ip, J. Luo and C.-J. Zhong, J. Phys. Chem. B, 2006, 110, 6673-6682.

7 N. T. Fofang, T.-H. Park, O. Neumann, N. A. Mirin, P. Nordlander and N. J. Halas, Nano Lett., 2008, 8, 3481-3487.

8 Y.-W. Hao, H.-Y. Wang, Y. Jiang, Q.-D. Chen, K. Ueno, W.-Q. Wang, H. Misawa and H.-B. Sun, Angew. Chem., Int. Ed., 2011, 50, 7824-7828.

9 W. H. Ni, H. J. Chen, J. Su, Z. H. Sun, J. F. Wang and H. K. Wu, J. Am. Chem. Soc., 2010, 132, 4806-4814.

10 W. H. Ni, Z. Yang, H. J. Chen, L. Li and J. F. Wang, J. Am. Chem. Soc., 2008, 130, 6692-6693.

11 W. Ni, T. Ambjornsson, S. P. Apell, H. Chen and J. Wang, Nano Lett., 2010, 10, 77-84.

12 A. M. Kelley, Nano Lett., 2007, 7, 3235-3240.

13 D. Gulen, J. Phys. Chem. C, 2010, 114, 13825-13831.

14 S. M. Morton and L. Jensen, J. Chem. Phys., 2011, 135, 134103.

15 P. L. Stiles, J. A. Dieringer, N. C. Shah and R. P. Van Duyne, Annu. Rev. Anal. Chem., 2008, 1, 601-626.

16 G. L. Liu, Y.-T. Long, Y. Choi, T. Kang and L. P. Lee, Nat. Methods, 2007, 4, 1015-1017.

17 J. R. Lakowicz, K. Ray, M. Chowdhury, H. Szmacinski, Y. Fu, J. Zhang and K. Nowaczyk, Analyst, 2008, 133, 1308-1346.

18 B. Dubertret, M. Calame and A. J. Libchaber, Nat. Biotechnol., 2001, 19, 365-370.

19 D. Cunningham, R. E. Littleford, W. E. Smith, P. J. Lundahl, I. Khan, D. W. McComb, D. Graham and N. Laforest, Faraday Discuss., 2006, 132, 135-145.

20 G. McNay, D. Eustace, W. E. Smith, K. Faulds and D. Graham, Appl. Spectrosc., 2011, 65, 825-837.

21 K. A. Willets, S. M. Stranahan and M. L. Weber, J. Phys. Chem. Lett., 2012, 3, 1286-1294.

22 I. A. Larmour, E. A. Argueta, K. Faulds and D. Graham, J. Phys. Chem. C, 2012, 116, 2677-2682.

23 X. Huang, S. Neretina and M. A. El-Sayed, Adv. Mater., 2009, 21, 4880-4910.

24 B. K. Juluri, M. Q. Lu, Y. B. Zheng, T. J. Huang and L. Jensen, J. Phys. Chem. C, 2009, 113, 18499-18503.

25 A. Yoshida, N. Uchida and N. Kometani, Langmuir, 2009, 25, 11802-11807.

26 G. von Maltzahn, A. Centrone, J.-H. Park, R. Ramanathan, M. J. Sailor, T. A. Hatton and S. N. Bhatia, Adv. Mater., 2009, 21, 3175-3180.

27 S. Pal, L. E. Depero and I. Alessandri, Nanotechnology, 2010, 21, 425701.

28 H. Cai, J. Zhu, G. Chen, L. Liu, G. S. He and X. Zhang, J. Raman Spectrosc., 2011, 42, 1722-1727.

29 A. M. Gabudean, M. Focsan and S. Astilean, J. Phys. Chem. C, 2012, 116, 12240-12249.

30 S. T. Sivapalan, B. M. DeVetter, T. K. Yang, T. van Dijk, M. V. Schulmerich, P. S. Carney, R. Bhargava and C. J. Murphy, ACS Nano, 2013, 7, 2099-2105.

31 A. McLintock, N. Hunt and A. W. Wark, Chem. Commun., 2011, 47, 3757-3759.

32 T. K. Sau and C. J. Murphy, Langmuir, 2004, 20, 6414-6420.

33 B. Nikoobakht and M. A. El-Sayed, Chem. Mater., 2003, 15, 1957-1962.
34 J. Turkevich, P. C. Stevenson and J. Hiller, Discuss. Faraday Soc., 1951, 11, 55.

35 W. Haiss, N. T. K. Thanh, J. Aveyard and D. G. Fernig, Anal. Chem., 2007, 79, 4215-4221.

36 A. M. Alkilany, R. L. Frey, J. L. Ferry and C. J. Murphy, Langmuir, 2008, 24, 10235-10239.

37 C. J. Orendorff and C. J. Murphy, J. Phys. Chem. B, 2006, 110, 3990-3994.

38 A. W. Wark, R. J. Stokes, S. B. Darby, W. E. Smith and D. Graham, J. Phys. Chem. C, 2010, 114, 18115-18120.

39 Y. B. Zheng, B. K. Juluri, L. L. Jensen, D. Ahmed, M. Lu, L. Jensen and T. J. Huang, Adv. Mater., 2010, 22, 3603-3607.

40 N. T. Fofang, N. K. Grady, Z. Fan, A. O. Govorov and N. J. Halas, Nano Lett., 2011, 11, 1556-1560.

41 H. Chen, L. Shao, K. C. Woo, J. Wang and H.-Q. Lin, J. Phys. Chem. C, 2012, 116, 14088-14095.

42 Y. B. Zheng, B. Kiraly, S. Cheunkar, T. J. Huang and P. S. Weiss, Nano Lett., 2011, 11, 2061-2065.

43 J. Zhao, J. A. Dieringer, X. Zhang, G. C. Schatz and R. P. Van Duyne, J. Phys. Chem. C, 2008, 112, 19302-19310.

44 K. L. Kelly, E. Coronado, L. L. Zhao and G. C. Schatz, J. Phys. Chem. B, 2003, 107, 668-677.

45 E. C. Le Ru, E. Blackie, M. Meyer and P. G. Etchegoin, J. Phys. Chem. C, 2007, 111, 13794-13803.

46 V. Amendola and M. Meneghetti, Adv. Funct. Mater., 2012, 22, 353-360.

47 J. R. Lombardi and R. L. Birke, Acc. Chem. Res., 2009, 42, 734-742.

48 J. C. S. Costa, R. A. Ando, P. H. C. Camargo and P. Corio, J. Phys. Chem. C, 2011, 115, 4184-4190.

49 C. Fernandez-Lopez, C. Mateo-Mateo, R. A. Alvarez-Puebla, J. Perez-Juste, I. Pastoriza-Santos and L. M. Liz-Marzan, Langmuir, 2009, 25, 13894-13899.

50 X. Su, J. Zhang, L. Sun, T.-W. Koo, S. Chan, N. Sundararajan, M. Yamakawa and A. A. Berlin, Nano Lett., 2005, 5, 49-54.

51 G. Goddard, L. O. Brown, R. Habbersett, C. I. Brady, J. C. Martin, S. W. Graves, J. P. Freyer and S. K. Doorn, J. Am. Chem. Soc., 2010, 132, 6081-6090.

52 T. A. Laurence, G. Braun, C. Talley, A. Schwartzberg, M. Moskovits, N. Reich and T. Huser, J. Am. Chem. Soc., 2009, 131, 162-169.

53 N. Pazos-Perez, C. S. Wagner, J. M. Romo-Herrera, L. M. LizMarzan, F. J. Garcia de Abajo, A. Wittemann, A. Fery and R. A. Alvarez-Puebla, Angew. Chem., Int. Ed., 2012, 51, 12688-12693.

54 H. Yuan, Y. Liu, A. M. Fales, Y. L. Li, J. Liu and V.-D. Tuan, Anal. Chem., 2013, 85, 208-212.

55 J. Song, J. Zhou and H. Duan, J. Am. Chem. Soc., 2012, 134, 13458-13469.

56 M. Sanles-Sobrido, W. Exner, L. Rodriguez-Lorenzo, B. Rodriguez-Gonzalez, M. A. Correa-Duarte, R. A. Alvarez-Puebla and L. M. Liz-Marzan, J. Am. Chem. Soc., 2009, 131, 2699-2705.

57 L. G. Xu, Y. Liu, Z. Y. Chen, W. Li, L. M. Wang, X. C. Wu, Y. L. Ji, Y. L. Zhao, L. Y. Ma, Y. M. Shao and C. Y. Chen, Nano Lett., 2012, 12, 2003-2012.

58 A. M. Alkilany, P. K. Nagaria, C. R. Hexel, T. J. Shaw, C. J. Murphy and M. D. Wyatt, Small, 2009, 5, 701-708. 